\title{
Double trouble: a patient with both HLA-B27 anterior uveitis and HLA-A29 birdshot chorioretinitis
}

\author{
Zeina Haddad ${ }^{*}$ and Ashvini Reddy
}

\begin{abstract}
Background: Birdshot chorioretinitis (BSCR) is a rare ocular inflammatory disorder associated with HLA-A29 and characterized by bilateral choroidal lesions, vitritis, macular edema, and retinal vasculitis. Ocular inflammation associated with HLA-B27 is typically a recurrent, unilateral, acute anterior uveitis (AAU) that is frequently associated with ankylosing spondylitis (AS). To date, there are no reports of patients with both HLA-A29-positive BSCR and HLA-B27 AAU/AS in the English literature.

Findings: A 50-year-old man with a history of bilateral anterior uveitis, vitritis, retinal vasculitis, and cream-colored depigmented oval choroidal lesions was found to be HLA-A29 and HLA-B27 positive. His lumbar spine and sacroiliac joint films revealed fusion of the spine, known as 'bamboo spine' compatible with the diagnosis of ankylosing spondyloarthropathy. He had chronic ocular inflammation that was difficult to control with systemic steroids and immunomodulatory agents.

Conclusions: This is the only report of a patient with both HLA-A29-positive BSCR and HLA-B27-positive AS and associated anterior uveitis. The severity of his disease suggests that patients who test positive for both HLA-A29 and HLA-B27 carry a poor visual prognosis. Prompt diagnosis and treatment with local or systemic corticosteroids or steroid-sparing agents may control the disease.
\end{abstract}

Keywords: Birdshot chorioretinitis; Ankylosing spondyloarthropathy; Seronegative spondyloarthropathy;

Panuveitis; Cystoid macular edema

\section{Findings Introduction}

Birdshot chorioretinitis (BSCR) is a rare ocular disorder characterized by bilateral choroidal lesions and chronic intraocular inflammation without anterior segment complications. A minimum of three discrete, round or oval, cream-colored foci of depigmentation is required for diagnosis. The lesions are most often one quarter to one half optic disc diameter in size and clustered around the disc, nearly always with involvement of the inferior and nasal peripapillary area. Exclusion criteria include keratic precipitates and posterior synechiae, which may form as a consequence of independent HLA-B27-related anterior uveitis [1].

\footnotetext{
* Correspondence: zah3q@hscmail.mcc.virginia.edu

Department of Ophthalmology, University of Virginia, 1300 Jefferson Park

Avenue, PO Box 800715, Charlottesville, VA 22908, USA
}

\section{Case description}

In 2002, a 38-year-old male sought care for flashes and floaters, blurred vision, intermittent eye redness, and photophobia OU. Initial best corrected visual acuity was 20/20 OU with bilateral peripheral field constriction, vitritis, and choroidal lesions. At that time, he was diagnosed with bilateral panuveitis with cystoid macular edema (CME) and started on $40 \mathrm{mg}$ oral prednisone daily and topical steroid and nonsteroidal anti-inflammatory drops. A laboratory workup included complete blood count, erythrocyte sedimentation rate, antinuclear antibodies, rapid plasma reagin, fluorescent treponemal antibody absorption test, angiotensin converting enzyme, chest X-ray, and toxoplasmosis serology - all of which were within normal limits. In 2004, his visual acuity deteriorated to 20/60

\section{空


OU. A diagnostic vitrectomy was performed in the left eye and was negative for malignancy and infection. Vision failed to improve, despite bilateral cataract surgery, due to persistent bilateral vitritis, episodes of anterior uveitis, $\mathrm{CME}$, and epiretinal membranes. He was treated only with steroid and nonsteroidal anti-inflammatory drops over this time period.

In 2008, he was seen in follow-up and noted to have persistent ocular inflammation. His vision was 20/50 OU. His slit lamp examination was notable for mild vitritis bilaterally and $1+$ haze. He had no cells or flare in his anterior chamber. Fundus examination was remarkable for bilateral optic nerve pallor, cream-colored depigmented oval choroidal lesions most numerous nasal to the optic disc, retinal vessel attenuation, epiretinal membranes, and moderate CME with attenuation of foveal contour. A diagnosis of BSCR was suspected. A repeat laboratory workup was ordered along with HLA typing. Complete blood count, erythrocyte sedimentation rate, $\mathrm{C}$ reactive protein, antinuclear antibodies, rheumatoid factor, rapid plasma reagin, fluorescent treponemal antibody absorption test, angiotensin converting enzyme, chest X-ray, tuberculosis skin test, and Lyme, toxocara, and toxoplasmosis serologies were within normal limits. His HLA typing was positive for HLAA29. He was started on prednisone $60 \mathrm{mg}$ daily and referred to a rheumatologist, who noted cervical spine abnormalities with abnormal gait and posture. X-rays showed partial ankylosis of the bilateral sacroiliac joints and lumbar spine syndesmophytes (Figure 1). HLA-B27 typing was subsequently positive, and he was diagnosed with BSCR and ankylosing spondylitis (AS). He was started on methotrexate $20 \mathrm{mg}$ weekly, but this failed to control his ocular inflammation. He was switched to mycophenolate mofetil $1,000 \mathrm{mg}$ daily, which was later increased to $1,500 \mathrm{mg}$ daily as prednisone was being tapered.

On his most recent examination in 2014, vision was 20/200 OD and 20/80 OS. His slit lamp examination was remarkable for rare cells in the anterior chamber OS and moderate vitritis with $2+$ haze OU. There had been progressive worsening of visual fields (Figure 2). Fundus examination revealed stable bilateral choroidal lesions, optic nerve pallor, retinal vessel attenuation, epiretinal membranes, and mild cystic edema with attenuation of foveal contour (Figures 3 and 4). The patient reported an allergy to fluorescein dye. Indocyanine green angiography revealed hypofluorescent dark spots corresponding to birdshot lesions (Figure 5). His antiinflammatory and rheumatic therapeutic regimen consisted of mycophenolate mofetil $500 \mathrm{mg}$ TID for ocular inflammation and Voltaren and Vicodin for pain related to his AS. Steroid implant or a second steroid-sparing agent was recommended given his progressive disease.

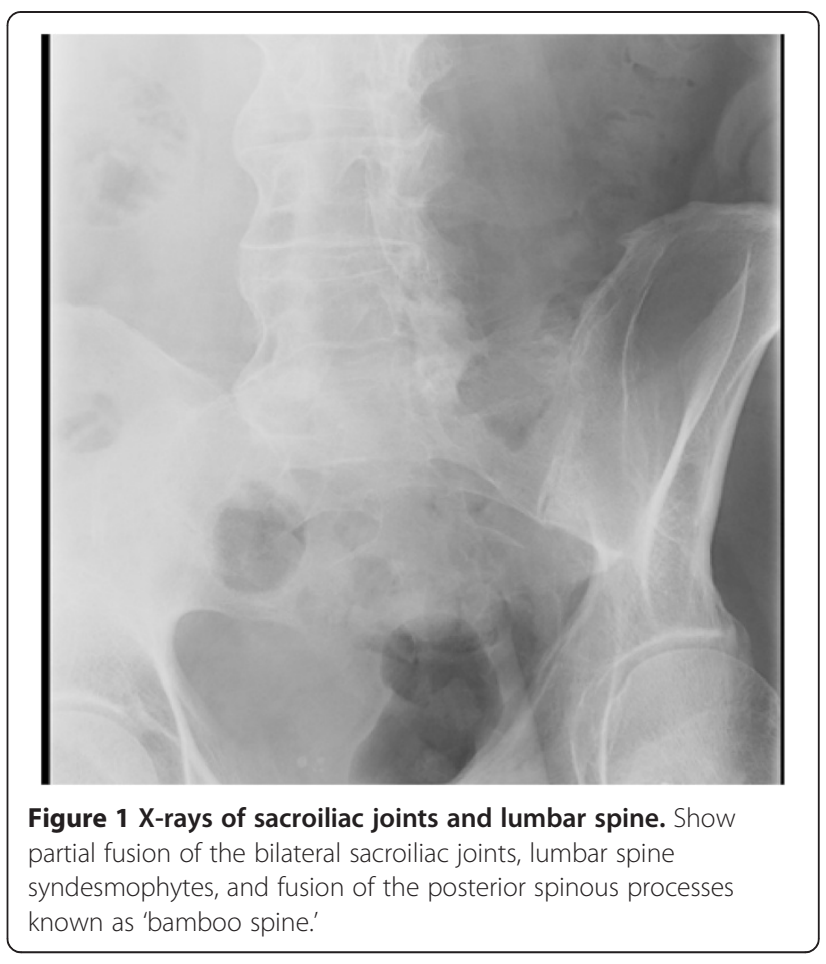

However, the patient declined any additional therapy and preferred close monitoring.

The patient provided consent for the report to be published.

\section{Discussion}

In 1975, Ryan and Maumenee [2] used the descriptive term birdshot retinochoroidopathy to define a rare ocular inflammatory disease characterized by bilateral creamcolored ovoid spots densest around the optic nerve and nasally.

Classically, eyes with BSCR are quiet with no ciliary injection or anterior chamber inflammation. Common posterior segment findings include hyperpermeable capillaries with CME, narrowing of retinal arterioles, perivascular hemorrhages, vessel tortuosity, and optic disc swelling. HLA typing is a valuable diagnostic tool but not a requirement for diagnosis; it has a sensitivity of $96 \%$ and a specificity of $93 \%$ [3].

The required characteristics for the diagnosis of BSCR according to Levinson [1] are bilateral disease, the presence of at least three peripapillary 'birdshot lesions' inferior or nasal to the optic disc in one eye, low-grade anterior segment intraocular inflammation (defined as $\leq 1+$ cells) and a low-grade vitreous inflammation (defined as $\leq 2+$ vitreous haze). Birdshot lesions are defined as cream-colored, irregular or elongated, choroidal lesions with indistinct borders, the long axis of which is radial to the optic disc. The ocular inflammatory activity was assessed according to the Standardization of Uveitis Nomenclature Working Group. 

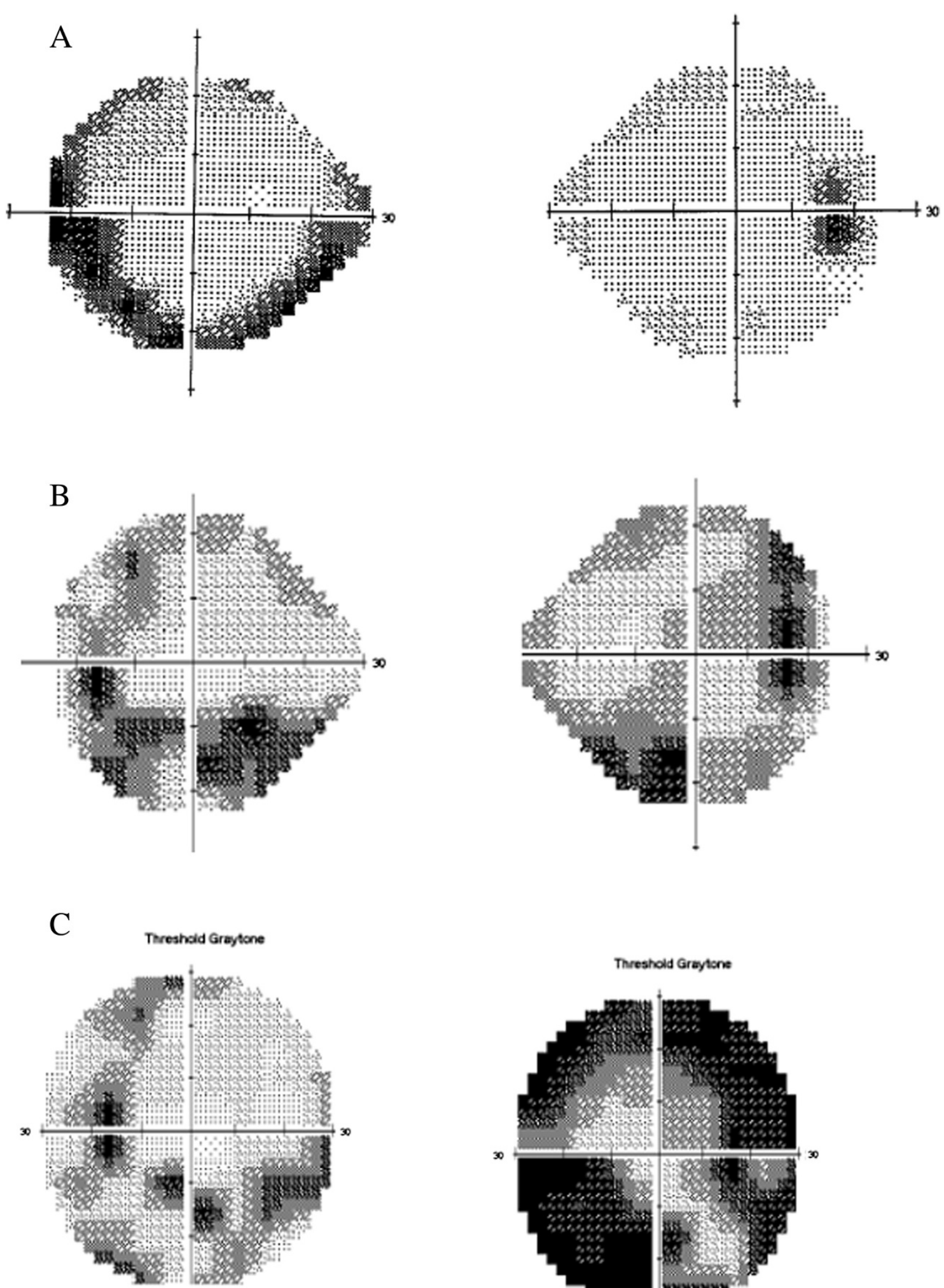

Figure 2 A-24-2 Humphrey visual fields done in 2002. Showing left inferior arcuate defect, enlarged blind spot, and few scattered desaturation points inferiorly in the right eye. B-24-2 Humphrey visual fields done in 2010 showing progressive worsening of visual field defect with bilateral inferior and superior arcuate defect more pronounced in the left eye with desaturation points around fixation in the right eye. C-30-2 Humphrey visual fields done in 2014 showing further progression of visual field defect with peripheral constriction and enlarged blind spot more pronounced in the right eye. 


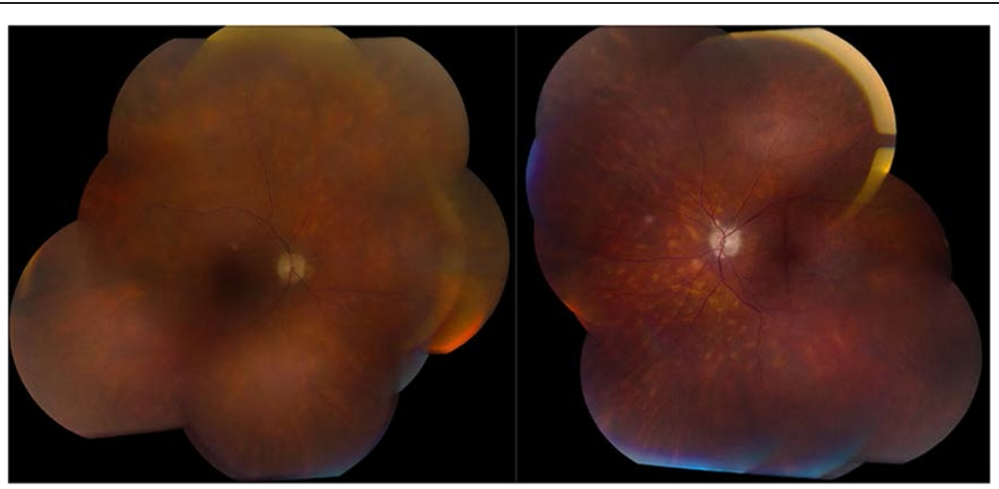

Figure 3 Montage fundus photos of the right and left eye. Showing cream-colored oval choroidal lesions scattered throughout the fundus.
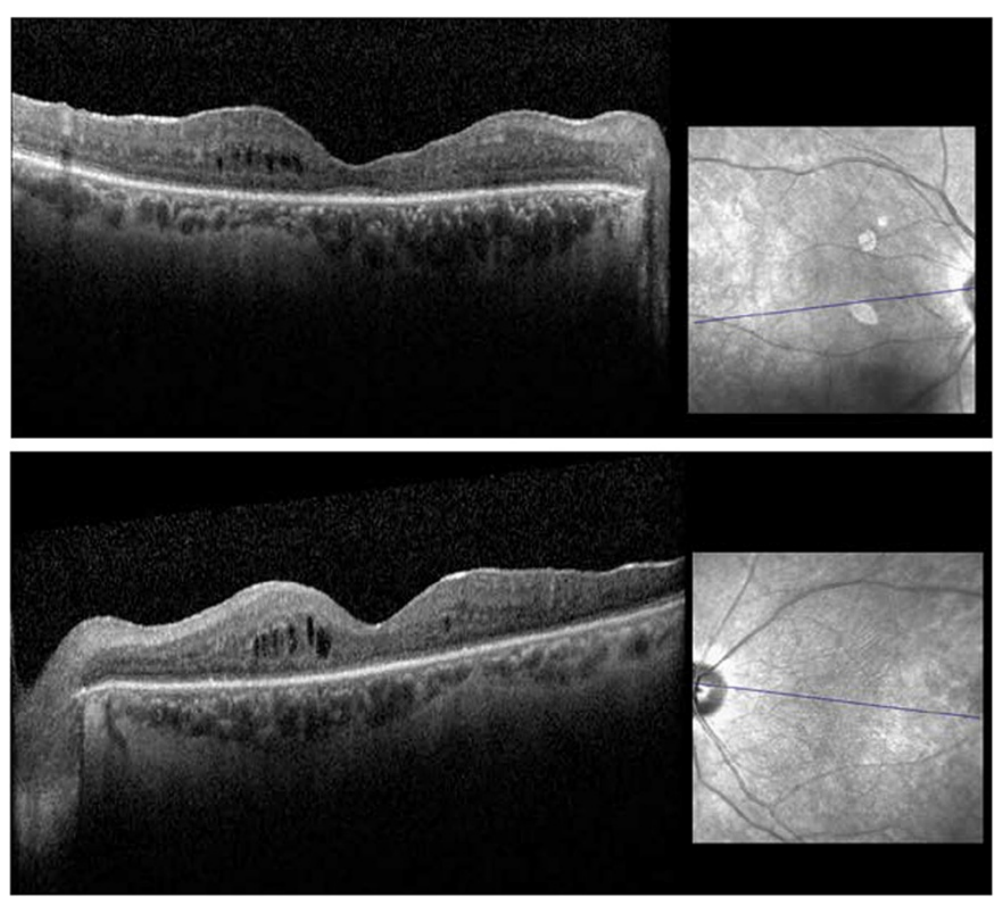

Figure 4 Spectral domain optical coherence tomography of the right and left eye. Showing epiretinal membranes, cystoid macular edema, and atrophic retina.

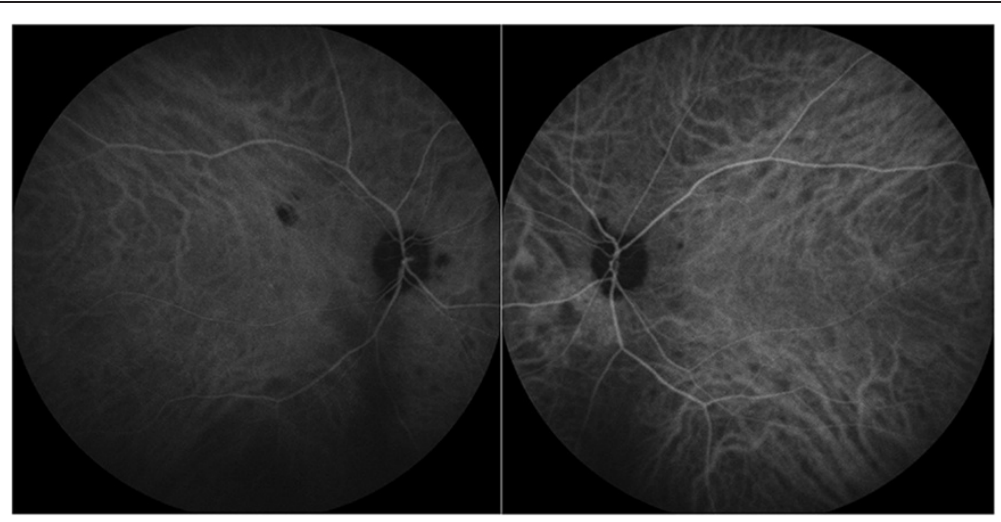

Figure 5 Indocyanine angiography revealed hypofluorescent areas corresponding to choroidal lesions. 
Supportive findings for the diagnosis of BSCR were: HLA-A29 positivity, retinal vasculitis, and CME. Exclusion criteria for the diagnosis of BSCR were the presence of keratic precipitates and posterior synechiae and the presence of infectious, neoplastic, or other inflammatory diseases that can cause multifocal choroidal lesions [1]. Exacerbations and remissions characterize the course of the disease. Loss of visual acuity is due to CME, macular epiretinal membrane formation, macular hole, subretinal neovascular membrane, macular scar, and cataract [4].

Our patient with AS-related acute anterior uveitis (AAU) was given a diagnosis of BSCR based on the clinical exam and had HLA typing confirming HLAA29 and HLA-B27 status. Over $90 \%$ of patients with AS are HLA-B27 positive, whereas only $6 \%$ to $8 \%$ of the general population is HLA-B27 positive. The primary ocular manifestation of AS is recurrent nongranulomatous acute anterior uveitis, which our patient reported. Acute anterior uveitis occurs in 25\% to $40 \%$ of patients with AS [5].

Priem and Oosterhuis reviewed 62 patients with BSCR. In half of the patients followed for 5 years or more, visual acuity was maintained at 20/60 or better [4]. More recent studies suggest that central visual acuity can be preserved long term in patients with BSCR. TomkinsNetzer et al. found that $88 \%(n=81)$ and $97 \%(n=89)$ of the eyes maintained best corrected visual acuity and did not progress to permanent visual loss $(<20 / 50)$ or severe visual loss $(<20 / 200)$, respectively over 10 years [6]. Our patient had uncontrolled ocular inflammation despite being on systemic steroids and immunomodulatory agents. Vision deteriorated to 20/200 OD and 20/80 OS with associated nerve pallor, epiretinal membranes, macular edema, and retinal atrophy. The severity of his disease suggests that patients who test positive for both HLA-A29 and HLA-B27 carry a poor visual prognosis, though delay to diagnosis and patient deferral of additional treatment are also contributing factors.

BSCR is generally considered to be an isolated ocular disorder. Few reports in the literature suggest a possible association with systemic illnesses including essential hypertension, cerebrovascular accidents, hearing loss, and cutaneous immune-mediated conditions such as vitiligo and psoriasis [4,7-10].

To our knowledge, this is the only reported case of a patient with both HLA-A29-positive BSCR and HLA-B27positive AS and associated anterior uveitis. Given the severity of his disease, we suspect that patients with this association develop inflammation that is difficult to control and have poorer visual prognosis than patients with BSCR alone. Prompt diagnosis and treatment with local or systemic corticosteroids or steroid-sparing agents may be of benefit.

\section{Abbreviations \\ AAU: acute anterior uveitis; AS: ankylosing spondylitis; BSCR: birdshot chorioretinitis; CME: cystoid macular edema.}

\section{Competing interests}

The authors declare that they have no competing interests.

\section{Authors' contributions}

$\mathrm{ZH}$ drafted the manuscript. AR reviewed and edited the final manuscript. AR provided ophthalmic care and managed the patient. Both the authors read and approved the final manuscript.

\section{Authors' information}

$\mathrm{ZH}$ is a third-year ophthalmology resident at the University of Virginia and had completed a two-year vitreoretinal fellowship at the University of Virginia. AR is a uveitis and a medical retina specialist at the University of Virginia.

\section{Acknowledgements}

The authors would like to acknowledge Drs. Kathryn Pepple, Paolo Silva, and Sophia Mirza Saleem who agreed to be peer reviewers for this manuscript.

Received: 2 July 2014 Accepted: 28 October 2014

Published online: 26 November 2014

\section{References}

1. Levinson RD, Brezin A, Rothova A, Accorinti M, Holland GN (2006) Research criteria for the diagnosis of birdshot chorioretinopathy: results of an international consensus conference. Am J Ophthalmol 141:185-187

2. Ryan SJ, Maumenee AE (1980) Birdshot retinochoroidopathy. Am J Ophthalmol 89:31-45

3. Feltkamp TEW (1990) Ophthalmological significance of HLA-associated uveitis. Eye 4:839-884

4. Priem HA, Oosterhuis JA (1988) Birdshot chorioretinopathy: clinical characteristics and evolution. Br J Ophthalmol 72:646-659

5. Van der Linden S, Van Der Heijde D (2001) Ankylosing spondylitis. In: Ruddy S, Harris ED, Sledge CB et al (eds) Kelly's textbook of rheumatology, 6th edn. Saunders, Philadelphia, pp 1039-1053

6. Tomkins-Netzer O, Taylor SR, Lightman S (2014) Long-term clinical and anatomic outcome of birdshot chorioretinopathy. JAMA 132(1):57-62

7. Gasch AT, Smith JA, Whitcup SM (1999) Birdshot retinochoroidopathy. Br J Ophthalmol 83(2):241-249

8. Gass JD (1981) Vitiliginous chorioretinitis. Arch Ophthalmol 99(10):1778-1787

9. Heaton JM, Mills RP (1993) Sensorineural hearing loss associated with birdshot retinochoroidopathy. Arch Otolaryngol Head Neck Surg 119(6):680-681

10. Rothova A, Berendschot TT, Probst K, van Kooij B, Baarsma GS (2004) Birdshot chorioretinopathy: long-term manifestations and visual prognosis. Ophthalmology 111(5):954-959

doi:10.1186/s12348-014-0028-6

Cite this article as: Haddad and Reddy: Double trouble: a patient with both HLA-B27 anterior uveitis and HLA-A29 birdshot chorioretinitis. Journal of Ophthalmic Inflammation and Infection 2014 4:28.

\section{Submit your manuscript to a SpringerOpen ${ }^{\odot}$ journal and benefit from:}

- Convenient online submission

- Rigorous peer review

- Immediate publication on acceptance

- Open access: articles freely available online

- High visibility within the field

- Retaining the copyright to your article

Submit your next manuscript at $>$ springeropen.com 